\title{
Baskakov operators and Jacobi weights: pointwise estimates
}

\author{
Jorge Bustamante ${ }^{1}$, Juan Jesús Merino-García² and José María Quesada ${ }^{3 *}$ (D)
}

${ }^{\text {*Correspondence: }}$

jquesada@ujaen.es

${ }^{3}$ Department of Mathematics, University of Jaén, Jaén, Spain

Full list of author information is available at the end of the article

\begin{abstract}
In this paper we present direct results (upper estimates) for Baskakov operators acting in spaces related with Jacobi-type weights. Our results include and extend some known facts related with this problem. The approach is based in the use of a new pointwise K-functional.
\end{abstract}

Keywords: Baskakov operators; Direct estimates; Jacobi weights; K-functionals

\section{Introduction}

Let $C[0, \infty)$ be the family of all real continuous functions on the semiaxis and $\mathcal{B}(0, \infty)$ the family of all bounded functions in $(0, \infty)$. A family of Baskakov operators is obtained as follows. For $\lambda \in \mathbb{R}, \lambda \geq 1$, and a function $f:[0, \infty) \rightarrow \mathbb{R}$, define (whenever the series converges)

$$
V_{\lambda}(f, x)=\sum_{k=0}^{\infty} f\left(\frac{k}{\lambda}\right) v_{\lambda, k}(x), \quad x \geq 0,
$$

where

$$
\nu_{\lambda, k}(x)=\left(\begin{array}{c}
\lambda+k-1 \\
k
\end{array}\right) \frac{x^{k}}{(1+x)^{\lambda+k}} .
$$

Some authors have considered these operators acting in spaces defined with the help of a Jacobi weight in the discrete case $(\lambda=n \in \mathbb{N})$ (see $[6,17,18]$, and [21]).

Let us present some notations. Throughout the paper we set $\varphi(x)=\sqrt{x(1+x)}$ and, for $x>0$,

$$
\varrho(x)=x^{a} /(1+x)^{b}
$$

where $a \geq-1$ and $b \in \mathbb{R}$ are fixed real parameters.

We will study approximation properties of the operators $V_{\lambda}$ in the weighted spaces of continuous functions

$$
C_{\varrho}[0, \infty)=\{f \in C[0, \infty): \varrho f \in \mathcal{B}(0, \infty)\}
$$

(c) The Author(s) 2021. This article is licensed under a Creative Commons Attribution 4.0 International License, which permits use, sharing, adaptation, distribution and reproduction in any medium or format, as long as you give appropriate credit to the original author(s) and the source, provide a link to the Creative Commons licence, and indicate if changes were made. The images or other third party material in this article are included in the article's Creative Commons licence, unless indicated otherwise in a credit line to the material. If material is not included in the article's Creative Commons licence and your intended use is not permitted by statutory regulation or exceeds the permitted use, you will need to obtain permission directly from the copyright holder. To view a copy of this licence, visit http://creativecommons.org/licenses/by/4.0/. 
When $0<a<1$ and $b>0$, it was proved in [21] that Baskakov operators are unbounded in $C_{\varrho}[0, \infty)$ with the usual weighted norm $\|\varrho f\|=\|\varrho f\|_{\infty}:=\sup _{x>0}|\varrho(x) f(x)|$. That is the reason why is better to consider the norm

$$
\|f\|_{\varrho}=|f(0)|+\sup _{x>0}|\varrho(x) f(x)| .
$$

To avoid complications, some authors prefer to work with the space

$$
C_{\varrho}^{0}[0, \infty)=\left\{f \in C_{\varrho}[0, \infty): f(0)=0\right\}
$$

For $\alpha \in[0,1]$, we also consider the space

$$
C_{\varrho}^{2, \alpha}:=C_{\varrho}^{2, \alpha}[0, \infty)=\left\{g \in C_{\varrho}[0, \infty): g^{\prime} \in A C_{\mathrm{loc}} \text { and } \varrho \varphi^{2 \alpha} g^{\prime \prime} \in \mathcal{B}(0, \infty)\right\}
$$

In this paper we present upper estimates for the error $\varrho(x)\left|f(x)-V_{\lambda}(f, x)\right|$ assuming that $a \geq-1$ and $b \in \mathbb{R}$.

In the discrete case, pointwise estimates have been obtained by some authors under more restrictive selections of the parameters $a$ and $b$. For instance, in the unweighted case $(a=b=0)$, Xie [20, Theorem 1.1] proved that, for $\alpha \in[0,1]$,

$$
\left|f(x)-V_{n}(f, x)\right| \leq C \omega_{\varphi^{\alpha}}^{2}\left(f, \frac{\varphi^{1-\alpha}(x)}{\sqrt{n}}\right), \quad f \in C[0, \infty) \cap \mathcal{B}(0, \infty),
$$

where $\omega_{\varphi^{\alpha}}^{2}(f, t)$ is the Ditzian-Totik modulus.

The case $\alpha=0$ was previously studied by Huo and Xue in [14, Theorem 1]. For $0<a<1$, $b>0$, and $\alpha \in[0,1]$, Wang and Xue [18, Theorem 1.1] verified the inequality

$$
\varrho(x)\left|f(x)-V_{n}(f, x)\right| \leq C \omega_{\varphi^{\alpha}}^{2}\left(f, \frac{\varphi^{1-\alpha}(x)}{\sqrt{n}}\right)_{\varrho}, \quad f \in C_{\varrho}[0, \infty) .
$$

The same result appeared in [17].

Our results are not restricted to the consideration of a larger family of parameters $a$ and $b$. There are two other important facts to be taken into account. First, instead of the classical $K$-functionals, we use a pointwise $K$-functional of the form

$$
K_{\alpha}(f, x, t)_{\varrho}=\inf _{g \in C_{\varrho}^{2, \alpha}}\left\{\|f-g\|_{\varrho}+t\left\|\varrho \varphi^{2} g^{\prime \prime}\right\|_{(0, x]}+t \varphi^{2(1-\alpha)}(x)\left\|\varrho \varphi^{2 \alpha} g^{\prime \prime}\right\|_{[x, \infty)}\right\} .
$$

In second place, we present the estimates for operators with the continuous parameter $\lambda$. Why are these two changes necessary? As we will show in another paper, the $K$-functional (3) is convenient for proving strong converse inequalities.

Notice that, when $\alpha=1$, the $K$-functional (3) is just equivalent to the usual one

$$
K_{\alpha}(f, t)_{\varrho}=\inf _{g \in C_{\varrho}^{2, \alpha}}\left\{\|f-g\|_{\varrho}+t\left\|\varrho \varphi^{2 \alpha} g^{\prime \prime}\right\|\right\}
$$

because, for all $x>0$,

$$
\left\|\varrho \varphi^{2} g^{\prime \prime}\right\| \leq\left\|\varrho \varphi^{2} g^{\prime \prime}\right\|_{(0, x]}+\left\|\varrho \varphi^{2} g^{\prime \prime}\right\|_{[x,+\infty)} \leq 2\left\|\varrho \varphi^{2} g^{\prime \prime}\right\| .
$$


Moreover, for $0 \leq \alpha \leq 1$ and $x>0$, we have

$$
K_{\alpha}(f, x, t)_{\varrho} \leq 2 K_{\alpha}\left(f, t \varphi^{2(1-\alpha)}(x)\right)_{\varrho} .
$$

Indeed, for $y \in(0, x], \alpha \in[0,1]$ and $g \in C_{\varrho}^{2, \alpha}$, we have

$$
\left|\varrho(y) \varphi^{2}(y) g^{\prime \prime}(y)\right|=\varphi^{2(1-\alpha)}(y)\left|\varrho(y) \varphi^{2 \alpha}(y) g^{\prime \prime}(y)\right| \leq \varphi^{2(1-\alpha)}(x)\left\|\varrho \varphi^{2 \alpha} g^{\prime \prime}\right\|
$$

and then

$$
\left\|\varrho \varphi^{2} g^{\prime \prime}\right\|_{(0, x]} \leq \varphi^{2(1-\alpha)}(x)\left\|\varrho \varphi^{2 \alpha} g^{\prime \prime}\right\|
$$

On the other hand, the use of a continuous parameter allows us to apply the results to study other family of operators. For a real $c>0, n>c$, and a function $f:[0, \infty) \rightarrow \mathbb{R}$, define

$$
W_{n, c}(f, x)=\sum_{k=0}^{\infty} f\left(\frac{k}{n}\right) w_{n, k, c}(x), \quad x \geq 0,
$$

whenever the series converges, where

$$
w_{n, k, c}(x)=\frac{C(n, k, c)}{k !} \frac{x^{k}}{(1+c x)^{k+n / c}}, \quad k>0
$$

with $C(n, k, c)=n(n+c) \cdots(n+(k-1) c)$, and $w_{n, 0, c}(x)=1 /(1+c x)^{n / c}$. Here $n$ is not necessarily an integer.

The operators (7) have been studied by several authors (see [3, 4, 10, 14], and [22]). Later we show that the operators $W_{n, c}(f, x)$ are related with Baskakov operators, but with a family of not integer parameters. This is a good reason for studying the operators $V_{\lambda}$.

The main results are given in Sect. 2 (pending of some auxiliary results that will be proved in Sect. 3). In Sect. 4 we explain how our approach can be used to obtain similar results for the operators (7). Converse results will appear in a separated paper.

The paper contains several references written in Chinese. We include them to provide a review in the topic. Since we do not use any result from these work, the reader can follow our arguments.

\section{Main results}

The inequality (8) was proved in [21, page 136], [15, Lemma 2.2] and [18, page 365-366] under the restriction $0<a<1$ and $b>0$. The same restrictions were considered in [10].

Proposition 2.1 Assume $a \geq-1, b \in \mathbb{R}, \alpha \in[0,1]$. If $0 \leq a+\alpha<2$, then there exists $a$ constant $C=C(a, b, \alpha)$ such that, for $\lambda>2(1+p)$, with $p=\max \{|b|, 2|b-\alpha|\}$ and $x>0$, one has

$$
\varrho(x) V_{\lambda}\left(\left|\int_{x}^{t} \frac{|t-u|}{\varrho(u) \varphi^{2 \alpha}(u)} d u\right|, x\right) \leq \frac{C}{\lambda} \varphi^{2(1-\alpha)}(x) .
$$


Proof By definition of $V_{\lambda}$,

$$
\varrho(x) V_{\lambda}\left(\left|\int_{x}^{t} \frac{|t-s|}{\varrho(s) \varphi^{2 \alpha}(s)} d s\right|, x\right)=\varrho(x) \sum_{k=0}^{\infty}\left|\int_{x}^{k / \lambda} \frac{|k / \lambda-s| d s}{\varrho(s) \varphi^{2 \alpha}(s)}\right| v_{\lambda, k}(x) .
$$

First we estimate in (i) the term corresponding to $k=0$, for $\lambda x \leq 1$. In (ii) we consider the sum for $k>0$ and $\lambda x \leq 1$. Finally, in part (iii) we estimate the sum in the case $\lambda x \geq 1$.

(i) Assume $k=0$ and $\lambda x \leq 1$. Then

$$
\varrho(x) \int_{0}^{x} \frac{s d s}{\varrho(s) \varphi^{2 \alpha}(s)} v_{\lambda, 0}(x)=\frac{x^{a}}{(1+x)^{b+\lambda}} \int_{0}^{x} s^{1-a-\alpha}(1+s)^{b-\alpha} d s .
$$

If $b \geq \alpha$, then from (9) we obtain

$$
\begin{aligned}
\varrho(x) \int_{0}^{x} \frac{s d s}{\varrho(s) \varphi^{2 \alpha}(s)} v_{\lambda, 0}(x) & \leq \frac{x^{a}}{(1+x)^{\alpha+\lambda}} \int_{0}^{x} s^{1-a-\alpha} d s \\
& =\frac{x^{2-\alpha}}{(2-a-\alpha)(1+x)^{\alpha+\lambda}}=\frac{x \varphi^{2(1-\alpha)}(x)}{(2-a-\alpha)(1+x)^{1+\lambda}} \\
& \leq \frac{\varphi^{2(1-\alpha)}(x)}{(2-a-\alpha) \lambda}
\end{aligned}
$$

If $b<\alpha$, then from (9) and the condition $b+\lambda \geq 0$, we get

$$
\begin{aligned}
\varrho(x) \int_{0}^{x} \frac{s d s}{\varrho(s) \varphi^{2 \alpha}(s)} v_{\lambda, 0}(x) & \leq x^{a} \int_{0}^{x} s^{1-a-\alpha} d s=\frac{x^{2-\alpha}}{2-a-\alpha} \\
& \leq \frac{x \varphi^{2(1-\alpha)}(x)}{(2-a-\alpha)(1+x)^{1-\alpha}} \leq \frac{\varphi^{2(1-\alpha)}(x)}{(2-a-\alpha) \lambda}
\end{aligned}
$$

(ii) Suppose $0<\lambda x \leq 1$ and $k>0$. If $b \geq 0$, then

$$
\begin{aligned}
\varrho(x) \int_{x}^{k / \lambda} \frac{(k / \lambda-s) d s}{\varrho(s) \varphi^{2 \alpha}(s)} & =\frac{x^{a}}{(1+x)^{b}} \int_{x}^{k / \lambda} \frac{(k / \lambda-s)(1+s)^{b} d s}{s^{a+\alpha}(1+s)^{\alpha}} \\
& \leq \frac{k / \lambda-x}{x^{\alpha}(1+x)^{b+\alpha}} \int_{x}^{k / \lambda} \frac{(1+s)^{\lceil b\rceil}}{(1+s)^{\lceil b\rceil-b}} d s \\
& \leq \frac{(k / \lambda-x)^{2}(1+k / \lambda)^{\lceil b\rceil}}{x^{\alpha}(1+x)^{\lceil b\rceil+\alpha}} .
\end{aligned}
$$

Hence, from Proposition 3.5,

$$
\begin{aligned}
& \varrho(x) \sum_{k=1}^{\infty} \int_{x}^{k / \lambda} \frac{(k / \lambda-s) d s}{\varrho(s) \varphi^{2 \alpha}(s)} v_{\lambda, k}(x) \\
& \leq \frac{1}{x^{\alpha}(1+x)^{\lceil b\rceil+\alpha}} \sum_{k=1}^{\infty}\left(\frac{k}{\lambda}-x\right)^{2}\left(1+\frac{k}{\lambda}\right)^{\lceil b\rceil} v_{\lambda, k}(x) \leq \frac{C}{x^{\alpha}(1+x)^{\alpha}} \frac{\varphi^{2}(x)}{\lambda} \\
& \quad=C \frac{\varphi^{2(1-\alpha)}(x)}{\lambda} .
\end{aligned}
$$


If $b<0$, we obtain

$$
\begin{aligned}
\varrho(x) \int_{x}^{k / \lambda} \frac{(k / \lambda-s) d s}{\varrho(s) \varphi^{2 \alpha}(s)} & =\frac{x^{a}}{(1+x)^{b}} \int_{x}^{k / \lambda} \frac{(k / \lambda-s)(1+s)^{b} d s}{s^{a+\alpha}(1+s)^{\alpha}} \\
& \leq \frac{1}{x^{\alpha}(1+x)^{\alpha}} \int_{x}^{k / \lambda}(k / \lambda-s) d s=\frac{(k / \lambda-x)^{2}}{2 x^{\alpha}(1+x)^{\alpha}}
\end{aligned}
$$

Then

$$
\begin{aligned}
\varrho(x) \sum_{k=1}^{\infty} \int_{x}^{k / \lambda} \frac{(k / \lambda-s) d s}{\varrho(s) \varphi^{2 \alpha}(s)} v_{\lambda, k}(x) & \leq \frac{1}{2 x^{\alpha}(1+x)^{\alpha}} \sum_{k=1}^{\infty}(k / \lambda-x)^{2} v_{\lambda, k}(x) \\
& \leq \frac{1}{2 x^{\alpha}(1+x)^{\alpha}} \frac{\varphi^{2}(x)}{\lambda}=\frac{\varphi^{2(1-\alpha)}(x)}{2 \lambda}
\end{aligned}
$$

(iii) Now we assume that $\lambda x \geq 1$. From Corollary 3.4 and Propositions 3.2 (i) and 3.6, one has $(C=1 /(2-a-\alpha))$

$$
\begin{aligned}
\varrho(x) & V_{\lambda}\left(\left|\int_{x}^{t} \frac{|t-u|}{u^{a+\alpha}(1+u)^{\alpha-b}} d u\right|, x\right) \\
\leq & C V_{\lambda}\left(\frac{(t-x)^{2}}{\varphi^{2 \alpha}(x)}\left(1+\frac{(1+t)^{b-\alpha}}{(1+x)^{b-\alpha}}\right), x\right) \\
& =\frac{C}{\varphi^{2 \alpha}(x)}\left(V_{\lambda}\left((t-x)^{2}, x\right)+\frac{V_{\lambda}\left((t-x)^{2}(1+t)^{b-\alpha}, x\right)}{(1+x)^{b-\alpha}}\right) \\
& \leq C\left(\frac{\varphi^{2(1-\alpha)}(x)}{\lambda}+\frac{\left(V_{\lambda}\left((t-x)^{4}, x\right)\right)^{1 / 2}\left(V_{\lambda}\left((1+t)^{2(b-\alpha)}, x\right)\right)^{1 / 2}}{\varphi^{2 \alpha}(x)(1+x)^{b-\alpha}}\right) \\
& \leq C\left(\frac{\varphi^{2(1-\alpha)}(x)}{\lambda}+C_{1} 2^{|b-\alpha|} \frac{\varphi^{2(1-\alpha)}(x)}{\lambda}\right) \leq C_{2} \frac{\varphi^{2(1-\alpha)}(x)}{\lambda} .
\end{aligned}
$$

Proposition 2.2 If $b \in \mathbb{R}, a \geq-1, \varrho(x)=x^{a} /(1+x)^{b}$, then there exists a constant $C=C(a, b)$ such that, for all $f \in C_{\varrho}[0, \infty), x>0$ and $\lambda \geq 2(1+p)$, with $p=|b|$, if $a=-1$, and $p=$ $\max \{2|a|, 2|b|,|b| /(1+a)\}$ otherwise, one has

$$
\varrho(x)\left|V_{\lambda}(f, x)\right| \leq C\|f\|_{\varrho}
$$

Proof If $f \in C_{\varrho}[0, \infty)$ and $x>0$, then

$$
\varrho(x)\left|V_{\lambda}(f, x)\right| \leq \frac{\varrho(x)|f(0)|}{(1+x)^{\lambda}}+\varrho(x) \sum_{k=1}^{\infty}\left|f\left(\frac{k}{\lambda}\right)\right| v_{\lambda, k}(x) .
$$

If $a<0$, then $f(0)=0$. If $a \geq 0$ (note that $\lambda+b \geq a$ ), then

$$
\frac{\varrho(x)|f(0)|}{(1+x)^{\lambda}}=\frac{x^{a}|f(0)|}{(1+x)^{\lambda+b}} \leq \frac{x^{a}|f(0)|}{(1+x)^{a}} \leq|f(0)| .
$$

On the other hand

$$
\varrho(x) \sum_{k=1}^{\infty}\left|f\left(\frac{k}{\lambda}\right)\right| v_{\lambda, k}(x) \leq \varrho(x)\|\varrho f\| \sum_{k=1}^{\infty} \frac{v_{\lambda, k}(x)}{\varrho(k / \lambda)} \leq C\|\varrho f\|,
$$


where in the last inequality we have used Proposition 3.8. Hence,

$$
\varrho(x)\left|V_{\lambda}(f, x)\right| \leq|f(0)|+C\|\varrho f\|=C_{1}\|f\|_{\varrho},
$$

where $C_{1}=\max \{1, C\}$.

Theorem 2.3 Assume $b \in \mathbb{R}, a \in[-1,1), \varrho(x)=x^{a} /(1+x)^{b}$ and $\alpha \in[0,1]$. If $0 \leq a+\alpha$, then there exist a constant $C=C(a, b, \alpha)$ and $\lambda_{0}>0$, such that, for $x>0, f \in C_{\varrho}[0, \infty)$ and $\lambda \geq \lambda_{0}$, one has

$$
\varrho(x)\left|f(x)-V_{\lambda}(f, x)\right| \leq C K_{\alpha}\left(f, x, \frac{1}{\lambda}\right)_{\varrho}
$$

where $K_{\alpha}(f, x, t)_{\varrho}$ is defined in (3).

Proof If $x>0$ and $g \in C_{\varrho}^{2, \alpha}[0, \infty)$, we use the representation

$$
g(t)=g(x)+g^{\prime}(x)(t-x)+\int_{x}^{t} g^{\prime \prime}(u)(t-u) d u .
$$

If $t<x$, then

$$
\left|\int_{x}^{t} g^{\prime \prime}(u)(t-u) d u\right| \leq\left\|\varrho \varphi^{2} g^{\prime \prime}\right\|_{(0, x]} \int_{t}^{x} \frac{|t-u| d u}{\varphi^{2}(u) \varrho(u)},
$$

and, if $t>x$, then

$$
\left|\int_{x}^{t} g^{\prime \prime}(u)(t-u) d u\right| \leq\left\|\varrho \varphi^{2 \alpha} g^{\prime \prime}\right\|_{[x, \infty)} \int_{x}^{t} \frac{|t-u| d u}{\varphi^{2 \alpha}(u) \varrho(u)} .
$$

Hence, from Proposition 2.1, we have

$$
\begin{aligned}
\varrho(x) & \left|V_{\lambda}(g, x)-g(x)\right| \\
\leq & \varrho(x) V_{\lambda}\left(\left|\int_{x}^{t} g^{\prime \prime}(u)(t-u) d u\right|, x\right) \\
\leq & \varrho(x)\left\|\varrho \varphi^{2} g^{\prime \prime}\right\|_{(0, x]} V_{\lambda}\left(\left|\int_{t}^{x} \frac{|t-u| d u}{\varphi^{2}(u) \varrho(u)}\right|, x\right) \\
& +\varrho(x)\left\|\varrho \varphi^{2 \alpha} g^{\prime \prime}\right\|_{[x, \infty)} V_{\lambda}\left(\left|\int_{x}^{t} \frac{|t-u| d u}{\varphi^{2 \alpha}(u) \varrho(u)}\right|, x\right) \\
\leq & \frac{C}{\lambda}\left(\left\|\varrho \varphi^{2} g^{\prime \prime}\right\|_{(0, x]}+\varphi^{2(1-\alpha)}(x)\left\|\varrho \varphi^{2 \alpha} g^{\prime \prime}\right\|_{[x, \infty)}\right) .
\end{aligned}
$$

Now, using standard arguments and Proposition 2.2, we have, for $x>0$,

$$
\begin{aligned}
& \varrho(x)\left|f(x)-V_{\lambda}(f, x)\right| \\
& \quad \leq \varrho(x)|f(x)-g(x)|+\varrho(x)\left|V_{\lambda}(g-f, x)\right|+\varrho(x)\left|g(x)-V_{\lambda}(g, x)\right| \\
& \quad \leq(1+C)\|f-g\|_{\varrho}+\varrho(x)\left|g(x)-V_{\lambda}(g, x)\right| .
\end{aligned}
$$


Thus, the result follows from (11), (12) and the definition of the $K$-functional given in (3).

Note that the condition $a<1$ in Theorem 2.3 is necessary in order to apply Proposition 2.1 with $\alpha=1$ to the right side integral in (10). However, writing

$$
\left|\int_{x}^{t} g^{\prime \prime}(u)(t-u) d u\right| \leq\left\|\varrho \varphi^{2 \alpha} g^{\prime \prime}\right\|\left|\int_{x}^{t} \frac{|t-u| d u}{\varphi^{2 \alpha}(u) \varrho(u)}\right|
$$

we only need the condition $0 \leq a+\alpha<2$ to apply Proposition 2.1 and conclude that

$$
\varrho(x)\left(V_{\lambda}(g, x)-g(x)\right) \leq \frac{C}{\lambda} \varphi^{2(1-\alpha)}(x)\left\|\varrho \varphi^{2 \alpha} g^{\prime \prime}\right\| .
$$

Remark 1 The inequality (13) was proved in [8, Lemma 6] for $\alpha=0, b \in \mathbb{R}$, and $a \in[-1,0]$. For $a=b=0$ the inequality (13) was also proved in [19, page 106-107]. The case $a=0$ and $b \in \mathbb{N}$ appeared in [4, Lemma 5] and [22, Lemma 1.6] with $\alpha=0$.

Taking into account (12), (13), Proposition 2.2 and the definition of the $K$-functional given in (4) we have immediately the following result.

Corollary 2.4 Assume $b \in \mathbb{R}, a \geq-1, \varrho(x)=x^{a} /(1+x)^{b}$ and $\alpha \in[0,1]$. If $0 \leq a+\alpha<2$, then there exist a constant $C=C(a, b, \alpha)$ and $\lambda_{0}>0$ such that, if $\lambda \geq \lambda_{0}, x>0$, and $f \in C_{\varrho}[0, \infty)$, then

$$
\varrho(x)\left|f(x)-V_{\lambda}(f, x)\right| \leq C K_{\alpha}\left(f, \frac{\varphi^{2(1-\alpha)}(x)}{\lambda}\right),
$$

where $K_{\alpha}(f, t)$ is the $K$-functional given in (4).

Remark 2 In [5, 11] and [16], direct results as in (14) were given with $a=b=0$. In $[15,18]$ and [21] the problem was studied with $0<a<1$ and $b>0$. In [9, Proposition 3.3] the authors considered weights of the form $\varrho(x)=x^{\beta_{0}} /(1+x)^{\beta_{0}+\beta_{\infty}}$, with $\beta_{0}, \beta_{\infty} \in[-1,0]$. Theorem 1.2 of [6] is also included in Corollary 2.4. In fact, in [6] for reals $r, s$ and $\lambda$ satisfying $0 \leq r<\lambda \leq 1$ and $s \geq 0$, the author considered the weight $\varrho^{*}(x)=x^{r} /(1+x)^{s}$ and the norm $\left\|\varrho^{*} \varphi^{2(1-\lambda)} f\right\|$. But this is equivalent to use the weight $\varrho(x)=x^{a} /(1+x)^{b}$, with $a=r+1-\lambda \geq 0$ and $b=s-1+\lambda$.

The operators $V_{\lambda}$, with $\lambda=n \in \mathbb{N}$, were considered in [14] and [19], with $a=b=0$. In [4] and [22] the case $a=0$ and $b \in \mathbb{N}$ was studied, while in [10] it was assumed that $0<a<1$ and $b>0$.

Notice that, for $\alpha \in[0,1]$,

$$
x^{\alpha / 2} \leq \varphi^{\alpha}(x) \leq 2^{\alpha / 2} x^{\alpha / 2}, \quad 0<x<1 .
$$

Thus $\varphi^{\alpha}$ satisfies condition (6.1.3) in [5] with $\beta(0)=\alpha / 2$. On the other hand

$$
\varrho(x) \sim x^{a} \quad \text { as } x \rightarrow 0+\text { and } \quad \varrho(x) \sim x^{a-b} \quad \text { as } x \rightarrow \infty .
$$

Hence condition (6.1.2) in [5] also holds with $\gamma(0)=a$ and $\gamma(\infty)=a-b$. 
As in [5, page 56], if $a=0$, define

$$
\omega_{\varphi^{\alpha}}^{2}(f, t)_{\varrho}=\sup _{0<h \leq t}\left\|\varrho \Delta_{h \varphi^{\alpha}}^{2} f\right\|
$$

where $\Delta_{s}^{2} f(x)=f(x+s)-2 f(x)+f(x-s)$.

If $a>0$, define

$$
\omega_{\varphi^{\alpha}}^{2}(f, t)_{\varrho}=\sup _{0<h \leq t}\left\|\varrho \Delta_{h \varphi^{\alpha}}^{2} f\right\|_{\left[t^{*}, \infty\right)}+\sup _{0<h \leq t^{*}}\left\|\varrho \vec{\Delta}_{h \varphi^{\alpha}}^{2} f\right\|_{\left[0,12 t^{*}\right]^{\prime}}
$$

where $\vec{\Delta}_{s}^{2} f(x)=f(x+2 s)-2 f(x+s)+f(x)$, and

$$
t^{*}=\left(2^{1+\alpha / 2}\right)^{2 /(2-\alpha)} t^{2 /(2-\alpha)} .
$$

It is well known (see [5, Th. 6.1.1] that there exist positive constants $C_{1}$ and $t_{0}$ such that, for $f \in C_{\varrho}^{0}[0, \infty)$ and $0<t \leq t_{0}$,

$$
\frac{1}{C_{1}} \omega_{\varphi^{\alpha}}^{2}(f, t)_{\varrho} \leq K_{\alpha}\left(f, t^{2}\right)_{\varrho} \leq C_{1} \omega_{\varphi^{\alpha}}^{2}(f, t)_{\varrho}
$$

where $K_{\alpha}(f, t)_{\varrho}$ is given by (4), and the modulus is defined by (15) when $a=0$ and by (16) when $a>0$.

For $a<0$, we do not know a characterization of the K-functional in terms of an appropriate modulus of smoothness.

From Corollary 2.4 and (17), we obtain the following result.

Theorem 2.5 Assume $b \in \mathbb{R}, a \in[0,1), \varrho(x)=x^{a} /(1+x)^{b}$ and $\alpha \in[0,1]$. There exist $a$ constant $C=C(a, b, \alpha)$ and $\lambda_{0}>0$ such that, if $\lambda \geq \lambda_{0}, x>0$ and $f \in C_{\varrho}^{0}[0, \infty)$, then

$$
\varrho(x)\left|f(x)-V_{\lambda}(f, x)\right| \leq C \omega_{\varphi^{\alpha}}^{2}\left(f, \frac{\varphi^{1-\alpha}(x)}{\sqrt{\lambda}}\right)_{\varrho}
$$

where $\omega_{\varphi^{\alpha}}^{2}(f, t)_{\varrho}$ is defined in (15) and (16).

In the discrete and unweighted case ( $n \in \mathbb{N}$ and $a=b=0)$, the inequality (18) was obtained by Huo and Xue [14, Theorem 1] in terms of the usual modulus of continuity $(\alpha=0)$ :

$$
\left|f(x)-V_{n}(f, x)\right| \leq 5 \omega_{2}\left(f, \sqrt{\frac{x(1+x)}{2 n}}\right) .
$$

In [20, Theorem 1.1], Xie proved an inequality of the form

$$
\left|f(x)-V_{n}(f, x)\right| \leq C \omega_{\varphi^{\gamma}}^{2}\left(f, \frac{\varphi^{1-\gamma}(x)}{\sqrt{n}}\right)
$$

any $\gamma \in[0,1]$.

For $0<a<1, b>0$ and $\alpha \in[0,1]$, the inequality (18) was obtained by Wang and Xue [18, Theorem 1.1]. Another proof was presented in [17]. 


\section{Auxiliary results}

Lemma 3.1 For $m \in \mathbb{N}, \lambda>m, x>0$ and $k \in \mathbb{N}_{0}$, one has

$$
\frac{1}{2^{m-1}}\left(\frac{1+k / \lambda}{1+x}\right)^{m} \leq \frac{v_{\lambda+m, k}(x)}{v_{\lambda, k}(x)} \leq\left(\frac{1+k / \lambda}{1+x}\right)^{m}
$$

Proof Note that for $k=0$ the inequality holds trivially. Next we will consider two cases:

Case I: Assume $0<k \leq m$. From the definition of $v_{\lambda, k}(x)$, we have,

$$
\begin{aligned}
\frac{v_{\lambda+m, k}(x)}{v_{\lambda, k}(x)} & =\frac{1}{(1+x)^{m}} \frac{(\lambda+m)(\lambda+m+1) \cdots(\lambda+k+m-1)}{\lambda(\lambda+1) \cdots(\lambda+k-1)} \\
& =\frac{1}{(1+x)^{m}} \prod_{j=0}^{k-1}\left(1+\frac{m}{\lambda+j}\right) .
\end{aligned}
$$

Then the result follows taking into account that, if $k<m$ and $\lambda>m$, then $1+k / \lambda \leq 1+$ $m / \lambda \leq 2$ and then

$$
\frac{1}{2^{m-1}}\left(1+\frac{k}{\lambda}\right)^{m} \leq 1+\frac{k}{\lambda} \leq 1+\frac{m}{\lambda} \leq \prod_{j=0}^{k-1}\left(1+\frac{m}{\lambda+j}\right) \leq\left(1+\frac{m}{\lambda}\right)^{k} \leq\left(1+\frac{k}{\lambda}\right)^{m}
$$

where in the last inequality we have applied that $(1+\beta p)^{q} \leq(1+\beta q)^{p}$ for $p, q \in \mathbb{N}_{0}, p \geq q$ and $\beta \geq 0$.

Case II: Now suppose $k>m$. In this case, we have

$$
\begin{aligned}
\frac{v_{\lambda+m, k}(x)}{v_{\lambda, k}(x)} & =\frac{1}{(1+x)^{m}} \frac{(\lambda+k)(\lambda+k+1) \cdots(\lambda+k+m-1)}{\lambda(\lambda+1) \cdots(\lambda+m-1)} \\
& =\frac{1}{(1+x)^{m}} \prod_{j=0}^{m-1}\left(1+\frac{k}{\lambda+j}\right)
\end{aligned}
$$

and then the result follows taking into account that, for $j=0,1, \ldots, m-1$,

$$
\frac{1}{2}\left(1+\frac{k}{\lambda}\right) \leq \frac{\lambda}{\lambda+j}\left(1+\frac{k}{\lambda}\right) \leq\left(1+\frac{k}{\lambda+j}\right) \leq 1+\frac{k}{\lambda}
$$

For $m=0,1,2, \ldots$, the central moment of order $m$ of the operator $V_{\lambda}$ is defined by

$$
S_{\lambda, m}(x)=V_{\lambda}\left(\left(e_{1}-x\right)^{m}, x\right)
$$

where $e_{1}$ is the function defined by $e_{1}(t)=t$.

Proposition 3.2 For $\lambda \geq 1$, one has (see [3]):

(i) $S_{\lambda, 0}(x)=1, S_{\lambda, 1}(x)=0$, and for $m \geq 1$,

$$
S_{\lambda, m+1}(x)=\frac{1}{\lambda} \varphi^{2}(x)\left(S_{\lambda, m}^{\prime}(x)+m S_{\lambda, m-1}(x)\right)
$$

(ii) For each $m \in \mathbb{N}$ there exists a constant $C_{m}$ such that, if $\lambda x \geq 1$, then $S_{\lambda, 2 m}(x) \leq$ $C_{m} \varphi^{2 m}(x) / \lambda^{m}$. 
In particular, from (20), we obtain $S_{\lambda, 2}(x)=\varphi^{2}(x) / \lambda$ and, for $\lambda x \geq 1$,

$$
\begin{aligned}
S_{\lambda, 4}(x) & =\frac{\varphi^{4}(x)}{\lambda^{2}}\left(3+\frac{6}{\lambda}+\frac{1}{\lambda x(1+x)}\right) \\
& \leq \frac{\varphi^{4}(x)}{\lambda^{2}}\left(9+\frac{\lambda}{1+\lambda}\right) \leq 10 \frac{\varphi^{4}(x)}{\lambda^{2}},
\end{aligned}
$$

because the function $\Psi(x)=1 /(x(1+x))$ decreases on $(0,+\infty)$.

Proposition 3.3 Assume $r \geq 0, m, q \in \mathbb{R}$ and $m-r+1>0$. Then, for $x>0$ and $t \geq 0$, one has

$$
\left|\int_{x}^{t} \frac{(t-u)^{m}}{u^{r}}(1+u)^{q} d u\right| \leq \frac{|t-x|^{m+1}}{(m-r+1) x^{r}}\left((1+x)^{q}+(1+t)^{q}\right) .
$$

Proof First note that

$$
\left|\int_{x}^{t} \frac{(t-u)^{m}}{u^{r}}(1+u)^{q} d u\right| \leq\left((1+x)^{q}+(1+t)^{q}\right)\left|\int_{x}^{t} \frac{|t-u|^{m}}{u^{r}} d u\right| .
$$

Now we estimate the last integral in the expression above. If $t<x$, then, putting $u-t=$ $\tau(x-t)$, we have

$$
\begin{aligned}
\left|\int_{x}^{t} \frac{|t-u|^{m}}{u^{r}} d u\right| & =\int_{t}^{x} \frac{(u-t)^{m}}{u^{r}} d u=(x-t)^{m+1} \int_{0}^{1} \frac{\tau^{m}}{((1-\tau) t+\tau x)^{r}} d \tau \\
& \leq \frac{(x-t)^{m+1}}{x^{r}} \int_{0}^{1} \tau^{m-r} d \tau=\frac{(x-t)^{m+1}}{(m-r+1) x^{r}} .
\end{aligned}
$$

If $x<t$, then (note that $m>-1+r \geq-1$ )

$$
\left|\int_{x}^{t} \frac{|t-u|^{m}}{u^{r}} d u\right|=\int_{x}^{t} \frac{(t-u)^{m}}{u^{r}} d u \leq \frac{1}{x^{r}} \int_{x}^{t}(t-u)^{m} d u=\frac{(t-x)^{m+1}}{(m+1) x^{r}}
$$

An inequality similar to (22) was proved in [8, Lemma 3] with the conditions $0 \leq r \leq 2$ and $m-r \geq 0$.

Corollary 3.4 Assume $a \geq-1, b \in \mathbb{R}, \varrho(x)=x^{a} /(1+x)^{b}$ and $\alpha \in[0,1]$. If $0 \leq a+\alpha<2$, then, for $x>0$ and $t \geq 0$,

$$
\varrho(x)\left|\int_{x}^{t} \frac{|t-u|}{\varrho(u) \varphi^{2 \alpha}(u)} d u\right| \leq \frac{(t-x)^{2}}{(2-a-\alpha) \varphi^{2 \alpha}(x)}\left(1+\frac{(1+t)^{b-\alpha}}{(1+x)^{b-\alpha}}\right) .
$$

Proof Since

$$
\left|\int_{x}^{t} \frac{|t-u|}{\varrho(u) \varphi^{2 \alpha}(u)} d u\right|=\left|\int_{x}^{t} \frac{|t-u|(1+u)^{b-\alpha}}{u^{a+\alpha}} d u\right|
$$

the result follows from Proposition 3.3 with $q=b-\alpha, m=1$ and $r=a+\alpha$. 
In particular, from (23) we have

$$
\varrho(x)\left|\int_{x}^{t} \frac{|t-u|}{\varrho(u) \varphi^{2 \alpha}(u)} d u\right| \leq \frac{2(t-x)^{2}}{(2-a-\alpha) \varphi^{2 \alpha}(x)},
$$

for $t>x$ and $b \leq \alpha$ or $t<x$ and $b \geq \alpha$.

It is well known (see [3]) that the operator $V_{\lambda}$ satisfies the relation

$$
V_{\lambda}((t-x) f(t), x)=\frac{\varphi^{2}(x)}{\lambda} V_{\lambda}^{\prime}(f(t), x) .
$$

Writing $1+t=(1+x)+(t-x)$ and applying (25), we have

$$
V_{\lambda}((1+t) f(t), x)=(1+x) V_{\lambda}(f(t), x)+\frac{\varphi^{2}(x)}{\lambda} V_{\lambda}^{\prime}(f(t), x) .
$$

Using (25) twice, it is easy to show that

$$
V_{\lambda}\left((t-x)^{2} f(t), x\right)=\frac{\varphi^{2}(x)}{\lambda}\left(\left(\frac{\varphi^{2}(x)}{\lambda} V_{\lambda}^{\prime}(f(t), x)\right)^{\prime}+V_{\lambda}(f(t), x)\right) .
$$

An inequality similar to (28) was proved by Becker [2, Lemma 6] and Zhang [22, Lemma 1.5] with a unspecific constant. We include here a simpler proof and give the optimal constant.

Proposition 3.5 Let $m \in \mathbb{N}$ and $\lambda \geq 1$. Then

$$
\frac{1}{(1+x)^{m}} V_{\lambda}\left((t-x)^{2}(1+t)^{m}, x\right) \leq C(m) \frac{\varphi^{2}(x)}{\lambda}, \quad x \geq 0
$$

where $C(m)=\left(1+m+m^{2}\right) m !$

Proof Denote $P_{m}(x)=V_{\lambda}\left((1+t)^{m}, x\right)$. It follows from (26) with $f(t)=(1+t)^{m}$ that

$$
P_{m+1}(x)=(1+x) P_{m}(x)+\frac{x(1+x)}{\lambda} P_{m}^{\prime}(x) .
$$

Since $P_{1}(x)=1+x$, we deduce by induction that $P_{m}(x)$ is a polynomial of degree $m$ which can be written as

$$
P_{m}(x)=\sum_{k=1}^{m} c_{m, k}(\lambda)(1+x)^{k} x^{m-k}
$$

with $c_{m, k}(\lambda) \geq 0, k=1, \ldots, m$.

In what follows we denote by $\ell(P)$ the leader coefficient of a polynomial $P$. From (29) we also obtain the recurrence relation

$$
\ell\left(P_{1}\right)=1, \quad \ell\left(P_{m+1}\right)=\left(1+\frac{m}{\lambda}\right) \ell\left(P_{m}\right)
$$


and this implies that

$$
\ell\left(P_{m}\right)=\prod_{j=0}^{m-1}\left(1+\frac{j}{\lambda}\right), \quad m \geq 1
$$

Applying (27) with $f(t)=(1+t)^{m}$, we get

$$
V_{\lambda}\left((t-x)^{2}(1+t)^{m}, x\right)=\frac{\varphi^{2}(x)}{\lambda}\left(\left(\frac{\varphi^{2}(x)}{\lambda} P_{m}^{\prime}(x)\right)^{\prime}+P_{m}(x)\right)=: \frac{\varphi^{2}(x)}{\lambda} Q_{m}(x) .
$$

Taking into account

$$
\begin{aligned}
Q_{m}(x) & =\left(\frac{\varphi^{2}(x)}{\lambda} P_{m}^{\prime}(x)\right)^{\prime}+P_{m}(x)=\frac{x(1+x)}{\lambda} P_{m}^{\prime \prime}(x)+\frac{1+2 x}{\lambda} P_{m}^{\prime}(x)+P_{m}(x) \\
& =\frac{x(1+x)}{\lambda} P_{m}^{\prime \prime}(x)+\frac{1+x}{\lambda} P_{m}^{\prime}(x)+\frac{x}{\lambda} P_{m}^{\prime}(x)+P_{m}(x)
\end{aligned}
$$

and (30), we deduce that $Q_{m}$ is also a polynomial of degree $m$ which can be written as

$$
Q_{m}(x)=\sum_{k=0}^{m} d_{m, k}(\lambda)(1+x)^{m-k} x^{k}
$$

with $d_{m, k}(\lambda) \geq 0, k=0,1, \ldots, m$.

Hence

$$
R_{m}(x):=\frac{Q_{m}(x)}{(1+x)^{m}}=\sum_{k=1}^{m} d_{m, k}(\lambda)\left(\frac{x}{1+x}\right)^{m-k}
$$

and then, for all $x \geq 0$,

$$
R_{m}^{\prime}(x)=\sum_{k=0}^{m-1} d_{m, k}(\lambda)(m-k)\left(\frac{x}{1+x}\right)^{m-k-1} \frac{1}{(1+x)^{2}} \geq 0
$$

Thus $R_{m}$ is increasing on $[0,+\infty)$ and then, for all $x \geq 0$,

$$
R_{m}(x)=\frac{Q_{m}(x)}{(1+x)^{m}} \leq \lim _{x \rightarrow+\infty} \frac{Q_{m}(x)}{(1+x)^{m}}=\ell\left(Q_{m}\right)
$$

Finally, for all $x \geq 0$,

$$
\frac{1}{(1+x)^{m}} V_{\lambda}\left((t-x)^{2}(1+t)^{m}, x\right)=\frac{\varphi^{2}(x)}{\lambda} \frac{Q_{m}(x)}{(1+x)^{m}} \leq \ell\left(Q_{m}\right)
$$

To finish the proof, it is sufficient to note that

$$
\ell\left(Q_{m}\right)=\left(\frac{m(m-1)}{\lambda}+\frac{2 m}{\lambda}+1\right) \ell\left(P_{m}\right)=\left(1+\frac{m(m+1)}{\lambda}\right) \prod_{j=0}^{m-1}\left(1+\frac{j}{\lambda}\right) .
$$


Putting $\lambda=1$ in the above expression, we obtain a upper bound which is valid for all $\lambda \geq 1$ and $x \geq 0$. Hence, we conclude that

$$
\frac{1}{(1+x)^{m}} V_{\lambda}\left((t-x)^{2}(1+t)^{m}, x\right) \leq C(m) \frac{\varphi^{2}(x)}{\lambda}, \quad x \geq 0, \lambda \geq 1
$$

where $C(m)=\left(1+m+m^{2}\right) m !$.

Using (30) and repeating the same arguments as above, it can be proved that, for all $\lambda \geq 1, x \geq 0$,

$$
\frac{1}{(1+x)^{m}} V_{\lambda}\left((1+t)^{m}, x\right)=\frac{P_{m}(x)}{(1+x)^{m}} \leq \ell\left(P_{m}\right)=\prod_{j=0}^{m-1}\left(1+\frac{j}{\lambda}\right) \leq m ! .
$$

In what follows we use a different approach to get an inequality similar to (32) which is valid for all $m \in \mathbb{R}$. We will consider the ceiling and floor functions of a number $z \in \mathbb{R}$ defined by

$$
\lceil z\rceil=\min \{r \in \mathbb{Z}: r \geq z\} \quad \text { and } \quad\lfloor z\rfloor=\max \{r \in \mathbb{Z}: r \leq z\} .
$$

Proposition 3.6 For $d \in \mathbb{R}, x>0$ and any $\lambda$ satisfying $\lambda \geq 2(1+|d|)$, one has

$$
V_{\lambda}\left((1+t)^{d}, x\right) \leq 2^{|d|}(1+x)^{d}
$$

Proof Note that the above inequality is trivial when $d=0$.

(i) Assume $d=m \in \mathbb{N}$. From (32) and taking into account that $\lambda>m$, we have

$$
\frac{1}{(1+x)^{m}} V_{\lambda}\left((1+t)^{m}, x\right) \leq \prod_{j=0}^{m-1}\left(1+\frac{j}{\lambda}\right) \leq 2^{m-1}
$$

(ii) For $d=-m, m \in \mathbb{N}$, we use the right side inequality in Lemma 3.1 by replacing $\lambda$ by $\lambda-m$ (note that $\lambda-m>m$ ), to obtain

$$
v_{\lambda, k}(x) \leq\left(\frac{1+\frac{k}{\lambda-m}}{1+x}\right)^{m} v_{\lambda-m, k}(x) \leq 2^{m}\left(\frac{1+k / \lambda}{1+x}\right)^{m} v_{\lambda-m, k}(x),
$$

where in the last inequality we have taken into account that $\lambda>2 m$ and then

$$
1+\frac{k}{\lambda-m} \leq \frac{\lambda}{\lambda-m}\left(1+\frac{k}{\lambda}\right) \leq 2\left(1+\frac{k}{\lambda}\right) .
$$

Therefore

$$
\begin{aligned}
V_{\lambda}\left((1+t)^{-m}, x\right) & =\sum_{k=0}^{\infty}(1+k / \lambda)^{-m} v_{\lambda, k}(x) \\
& \leq 2^{m}(1+x)^{-m} \sum_{k=0}^{\infty} v_{\lambda-m, k}(x)=2^{m}(1+x)^{-m} .
\end{aligned}
$$


(iii) If $d>0$ and $d \notin \mathbb{N}$, then, applying Hölder's inequality and using (33), we have (note that $\lambda \geq 2(1+|d|)>2\lceil d\rceil)$,

$$
V_{\lambda}\left((1+t)^{d}, x\right) \leq\left(V_{\lambda}\left((1+t)^{\lceil d\rceil}, x\right)\right)^{d /\lceil d\rceil} \leq 2^{(\lceil d\rceil-1) d /\ulcorner d\rceil}(1+x)^{d} \leq 2^{d}(1+x)^{d} .
$$

Similarly, if $d<0$ and $-d \notin \mathbb{N}$, we apply again Hölder's inequality and (34) (note that $\lambda \geq$ $2(1+|d|) \geq 2|\lfloor d\rfloor|$ and $0<d /\lfloor d\rfloor)<1)$, to obtain

$$
\left.V_{\lambda}\left((1+t)^{d}, x\right) \leq V_{\lambda}(1+t)^{\lfloor d\rfloor}, x\right)^{d /\lfloor d\rfloor} \leq 2^{|\lfloor d\rfloor| d /\lfloor d\rfloor}(1+x)^{d}=2^{|d|}(1+x)^{d} .
$$

A result similar to Proposition 3.7 was proved in [10, Lemma 1] in the case that $\lambda=n \in \mathbb{N}$ and $d \in \mathbb{N}$, but with an unspecific constant. The arguments presented here are similar to the one given in [10]. Anyway we present a detailed proof because the quoted paper is not easy to obtain.

Proposition 3.7 For $\ell>0, x>0$ and any real $\lambda$ satisfying $\lambda \geq 2(1+\ell)$, one has

$$
\sum_{k=1}^{\infty}\left(\frac{\lambda}{k}\right)^{\ell} v_{\lambda, k}(x) \leq \frac{2^{\ell}(1+\lceil\ell\rceil) !}{x^{\ell}}
$$

Proof (i) Assume $\ell \in \mathbb{N}$. First note that, for $x>0$,

$$
\begin{aligned}
x^{\ell}\left(\frac{\lambda}{k}\right)^{\ell} \frac{v_{\lambda, k}(x)}{v_{\lambda-\ell, k+\ell}(x)} & =\left(\frac{\lambda}{k}\right)^{\ell} \frac{(k+\ell) !}{k !} \frac{\lambda(\lambda+1) \cdots(\lambda+k-1)}{(\lambda-\ell)(\lambda-\ell+1) \cdots(\lambda-\ell+(k+\ell-1))} \\
& =\left(\frac{\lambda}{k}\right)^{\ell} \frac{(k+\ell) !}{k !} \frac{1}{(\lambda-1)(\lambda-2) \cdots(\lambda-\ell)} \\
& =\frac{1}{k^{\ell}} \prod_{i=1}^{\ell}(k+i) \prod_{j=1}^{\ell}\left(1-\frac{j}{\lambda}\right)^{-1} .
\end{aligned}
$$

Also, note that

$$
\frac{1}{k^{\ell}} \prod_{i=1}^{\ell}(k+i)=\prod_{i=1}^{\ell}\left(1+\frac{i}{k}\right) \leq(1+\ell) !
$$

On the other hand, since $\lambda>2 \ell$, we have

$$
\prod_{j=1}^{\ell}\left(1-\frac{j}{\lambda}\right)^{-1} \leq\left(1-\frac{\ell}{\lambda}\right)^{-\ell}=\left(\frac{\lambda}{\lambda-\ell}\right)^{\ell}<2^{\ell}
$$

Therefore

$$
\sum_{k=1}^{\infty}\left(\frac{\lambda}{k}\right)^{\ell} v_{\lambda, k}(x) \leq \frac{2^{\ell}(1+\ell) !}{x^{\ell}} \sum_{k=1}^{\infty} v_{\lambda-\ell, k+\ell}(x) \leq \frac{2^{\ell}(1+\ell) !}{x^{\ell}} .
$$


(ii) Finally assume $\ell>0$ and $\ell \notin \mathbb{N}$. Using Hölder's inequality and (36), we have (note that $\lambda \geq 2(1+\ell) \geq 2\lceil\ell\rceil)$,

$$
\sum_{k=1}^{\infty}\left(\frac{\lambda}{k}\right)^{\ell} v_{\lambda, k}(x) \leq\left(\sum_{k=1}^{\infty}\left(\frac{\lambda}{k}\right)^{\lceil\ell\rceil} v_{\lambda, k}(x)\right)^{\ell / \Gamma \ell\rceil} \leq \frac{2^{\ell}(1+\lceil\ell\rceil) !}{x^{\ell}} .
$$

Proposition 3.8 For $a \in[-1, \infty)$ and $b \in \mathbb{R}$, there exists a constant $C=C(a, b)$ such that, for $x>0$ and $\lambda \geq 2(1+p)$, with $p=|b|$, if $a=-1$, and $p=\max \{2|a|, 2|b|,|b| /(1+a)\}$ otherwise, we have

$$
\sum_{k=1}^{\infty}\left(\frac{\lambda}{k}\right)^{a}\left(1+\frac{k}{\lambda}\right)^{b} v_{\lambda, k}(x) \leq C \frac{(1+x)^{b}}{x^{a}}, \quad x>0 .
$$

Proof When $a \geq 0$, we use Hölder's inequality to obtain

$$
\sum_{k=1}^{\infty}\left(\frac{\lambda}{k}\right)^{a}\left(1+\frac{k}{\lambda}\right)^{b} v_{\lambda, k}(x) \leq\left(\sum_{k=1}^{\infty}\left(\frac{\lambda}{k}\right)^{2 a} v_{\lambda, k}(x)\right)^{1 / 2}\left(V_{\lambda}\left((1+t)^{2 b}, x\right)\right)^{1 / 2},
$$

and then the result follows from Propositions 3.6 and 3.7.

When $-1<a<0$, we use again Hölder's inequality and Proposition 3.6 to get

$$
\begin{aligned}
\sum_{k=1}^{\infty}\left(\frac{k}{\lambda}\right)^{-a}\left(1+\frac{k}{\lambda}\right)^{b} v_{\lambda, k}(x) & \leq\left(V_{\lambda}(t, x)\right)^{-a}\left(V_{\lambda}\left((1+t)^{b /(1+a)}, x\right)\right)^{1+a} \\
& \leq 2^{|b|} \frac{(1+x)^{b}}{x^{a}}
\end{aligned}
$$

When $a=-1$ a different argument is needed. Note that, for $k \geq 1$, we have

$$
\frac{v_{\lambda, k}(x)}{v_{\lambda+1, k-1}(x)}=\frac{\lambda x}{k}
$$

and then

$$
\begin{aligned}
& \sum_{k=1}^{\infty} \frac{k}{\lambda}\left(1+\frac{k}{\lambda}\right)^{b} v_{\lambda, k}(x) \\
& \quad=x \sum_{k=1}^{\infty}\left(1+\frac{k}{\lambda}\right)^{b} v_{\lambda+1, k-1}(x) \\
& \quad=x \sum_{k=0}^{\infty}\left(1+\frac{k+1}{\lambda}\right)^{b} v_{\lambda+1, k}(x)=x\left(\frac{\lambda+1}{\lambda}\right)^{b} \sum_{k=0}^{\infty}\left(1+\frac{k}{\lambda+1}\right)^{b} v_{\lambda+1, k}(x) \\
& \quad \leq 2^{2|b|} x(1+x)^{b},
\end{aligned}
$$

where in the last inequality we have applied Proposition 3.6 and the fact that $1 \leq \frac{\lambda+1}{\lambda} \leq 2$.

\section{Application to other operators}

As we remark in the Introduction, the operators $W_{n, c}$, with $c>0$ and $n>c$ have been studied by some authors. Some estimates for these operators can be inferred by the one 
presented above. In fact, if $f:[0, \infty) \rightarrow \mathbb{R}$ and we set $g(t)=f(t / c)$, then

$$
\begin{aligned}
V_{n / c}(g, c x) & =\sum_{k=0}^{\infty} g\left(\frac{c k}{n}\right)\left(\begin{array}{c}
n / c+k-1 \\
k
\end{array}\right) \frac{(c x)^{k}}{(1+c x)^{k+n / c}} \\
& =\sum_{k=0}^{\infty} f\left(\frac{k}{n}\right) \frac{n(n+c) \cdots(n+(k-1) c)}{k !} \frac{x^{k}}{(1+c x)^{k+n / c}} \\
& =\sum_{k=0}^{\infty} f\left(\frac{k}{n}\right) \frac{C(n, k, c)}{k !} \frac{x^{k}}{(1+c x)^{k+n / c}}=W_{n, c}(f, x) .
\end{aligned}
$$

For the operators $W_{n, c}$, Hou and Xue [14] gave upper estimates in the unweighted case $(a=b=0)$. Chen and Wu in [4] and by Zhang in [22] considered the same operators but acting in weighted spaces with polynomial weights $(a=0$ and $b \in \mathbb{N})$. While Gao, Kong and Xue [10] analyzed the weight $\varrho(x)=x^{a} /(1+x)^{b}$, with $0<a<1$ and $b>0$. All the results of these authors can be recovered for our approach by using equation (37).

The ideas in this work can be used to study other families of operators like Lupas, SzászMirakyan and some modifications of Baskakov operators, like the ones considered in [1, $7,12,13]$. For instance, in [13] only some special polynomial weights were presented.

In our setting, for the case of Szász-Mirakyan operators, the authors will present similar results in a forthcoming work.

\section{Acknowledgements}

The second and the third authors are partially supported by Junta de Andalucía Research Group FQM268. Also the third author is partially supported by Research Project PGC2018-097621-B-100 and EI-FQM08-2019.

\section{Funding}

Not applicable.

Availability of data and materials

Not applicable.

Competing interests

The authors declare that they have no competing interests.

\section{Authors' contributions}

The authors worked together in this project. Also, the final manuscript was read and approved for all the authors.

\section{Author details}

${ }^{1}$ Facultad de Ciencias Físico-Matemáticas, Benemérita Universidad Autónoma de Puebla, Puebla, México. ${ }^{2}$ IES. Juan Ramón Jiménez. Moguer, Huelva, Spain. ${ }^{3}$ Department of Mathematics, University of Jaén, Jaén, Spain.

\section{Publisher's Note}

Springer Nature remains neutral with regard to jurisdictional claims in published maps and institutional affiliations.

Received: 24 February 2021 Accepted: 23 June 2021 Published online: 13 July 2021

\section{References}

1. Abel, U., Gupta, V: An estimate of the rate of convergence of a Bezier variant of the Baskakov-Kantorovich operators for bounded variation functions. Demonstr. Math. 36(1), 123-136 (2003)

2. Becker, M.: Global approximation theorems for Szász-Mirakjan and Baskakov operators in polynomial weight spaces. Indiana Univ. Math. J. 27, 127-142 (1978)

3. Bustamante, J., Carrillo-Zentella, A., Quesada, J.M.: Direct and strong converse theorems for a general sequence of positive linear operators. Acta Math. Hung. 136(1-2), 90-106 (2012)

4. Chen, W., Wu, J.: The global approximation theorem for generalized Lupas-Baskakov operators. J. Xiamen Univ. Natur. Sci. 33(5), 571-576 (1994) (in Chinese)

5. Ditzian, Z., Totik, V.: Moduli of Smoothness. Springer, New York (1987)

6. Feng, G.: Direct and inverse approximation theorems for Baskakov operators with the Jacobi-type weight. Abstr. Appl. Anal. 2011, Article ID 101852 (2011) 
7. Finta, Z., Gupta, V.: Approximation properties of q-Baskakov operators. Cent. Eur. J. Math. 8(1), 199-211 (2010)

8. Gadjev, I.: Weighted approximation by Baskakov operators. Math. Inequal. Appl. 18(4), 1443-1461 (2015)

9. Gadjev, I., Parvanov, P.E.: Weighted approximation of functions in $L_{\infty}[0, \infty)$. Mediterr. J. Math. 14(6), 220: 14 pag (2017)

10. Gao, Y., Kong, N., Xue, Y.: Approximation with weights by generalized Baskakov operators. J. Ningxia Univ. (Nat. Sci.) 25(3), 196-200 (2004) (in Chinese)

11. Guo, S. Li, C., Zhang, G.: Pointwise estimate for Baskakov operators. Northeast. Math. J. 17(2), 133-137 (2001)

12. Gupta, V:: An estimate on the convergence of Baskakov-Bézier operators. J. Math. Anal. Appl. 312(1), 280-288 (2005)

13. Gupta, V., Acu, A.M., Sofonea, D.F.: Approximation of Baskakov type Pólya-Durrmeyer operators. Appl. Math. Comput. 294, 318-331 (2017)

14. Hou, X., Xue, Y.: Approximation by generalized Baskakov operators. Chin. Q. J. Math. 6(1-2), 115-121 (1990) (in Chinese)

15. Li, C:: The pointwise result on weighted approximation for Baskakov operators. J. Sichuan Univ. Natur. Sci. Ed. 37(1), 33-38 (2000) (in Chinese)

16. Totik, V.: Uniform approximation by Baskakov and Meyer-König and Zeller operators. Period. Math. 14, 209-228 (1983)

17. Wang, J.J., Xu, Z: Approximation with Jacobi weights by Baskakov operators. Taiwanese J. Math., 13(1), 157-168 (2009)

18. Wang, J.J., Xue, Y.: Direct and inverse approximation theorems with Jacobi weight for Baskakov operators and their derivatives. Chin. Ann. Math., Ser. A 26(4), 561-570 (2005) (in Chinese)

19. Wang, L., Xue, Y.: Generalized Baskakov operators and the direct and inverse theorem of its derivatives. J. Ningxia Univ. (Nat. Sci.) 23(2), 105-107 (2002) (in Chinese)

20. Xie, L.S.: Direct and inverse approximation theorems and derivatives of Baskakov operators. Chin. Ann. Math. 21A(3), 253-260 (2000) (in Chinese)

21. Xuan, P., Zhou, D.: Rate of convergence for Baskakov operators with Jacobi-weights. Acta Math. Appl. Sin. 18(1), 129-139 (1995) (in Chinese)

22. Zhang, X:: On character of global weighted approximation for general Baskakov operators in polynomial weighted spaces. J. Ningxia Univ. (Nat. Sci.) 12, 6-12 (1996) (in Chinese)

\section{Submit your manuscript to a SpringerOpen ${ }^{\circ}$ journal and benefit from:}

- Convenient online submission

Rigorous peer review

- Open access: articles freely available online

- High visibility within the field

- Retaining the copyright to your article

Submit your next manuscript at $\gg$ springeropen.com 